\title{
Evaluation of heart dysfunction in patients with rheumatoid arthritis
}

\author{
Nayyereh Saadati ${ }^{* *}$, Mahsa Moosavi ${ }^{2}$ \\ ${ }^{1}$ Ghaem Hospital Medical Center, Mashhad University of Medical Sciences, Mashhad, Iran; ${ }^{2}$ Neyshabur University of Medical \\ Sciences, Neyshabur, Iran
}

\begin{abstract}
Cardiovascular diseases are the most common causes of death in patients diagnosed with rheumatoid arthritis (RA). The high prevalence of cardiovascular disease is independent of known risk factors in RA patients. The present study aimed to estimate the left ventricular diastolic function in patients suffering from RA using conventional Doppler and tissue Doppler echocardiographic imaging (TDI). This descriptive, cross-sectional study was conducted on 40 patients who had an established diagnosis of RA for over five years $(2,7,9)$ and referred to the Rheumatology Department and Clinic of Ghaem Hospital, affiliated with Mashhad University of Medical Sciences. Patients with normal electrocardiography, chest examination, and chest radiography were subjected to M-mode, two-dimensional Doppler echocardiography. Data analysis was performed with the SPSS statistical software package (version 11.5). A p-value less than 0.05 was considered statistically significant. According to the results, mitral and tricuspid insufficiencies were the most common valvular diseases ( $72.5 \%$ and $52 \%$, respectively). In $70 \%$ of the patients, diastolic function was normal. A significant relationship was observed between systolic function and diastolic abnormality $(P=0.001)$. Pulmonary hypertension was detected in $40 \%$ of patients. Nevertheless, no significant relationship was observed between $\mathrm{E}$ and $\mathrm{Em}$ or between $\mathrm{A}$ and $\mathrm{Am}(P=0.432, P=0.844$, respectively). The Mann-Whitney $\mathrm{U}$ test revealed a statistical difference between the two groups in terms of EF, which was significantly lower in the diastolic dysfunction group $(P=0.047)$. A wide and frequent variety of cardiac diseases, especially mitral, aortic, and tricuspid regurgitation, may be found in RA patients. It can be concluded that active RA patients have a significantly high incidence of diastolic dysfunction in the absence of clinical evidence of heart disease.
\end{abstract}

Keywords: diastolic dysfunction, doppler findings, echocardiography, heart disease, rheumatoid arthritis.

\section{Introduction}

Rheumatoid arthritis (RA) is a systemic disorder associated with the involvement of several organs. The prevalence rate of this disease is about $1-2 \%$, and it is 2.5 times more common in women than men. Aging is reported to increase the prevalence of RA and its complications [1,2]. According to various studies, cardiovascular diseases are the main causes of mortality in patients with RA, the risk of which doubles with aging [2]. Megha Prasad et al. demonstrated that the high risk of cardiovascular problems in patients with RA is independent from the known risk factors for cardiovascular diseases [1]. Common cardiovascular problems of RA patients include pericarditis, atherosclerosis, myocarditis, congestive heart failure, and heart block; RA patients are also at high risk for valve involvement due to chronic valvulitis [2-4].
Patients with RA are at high risk for cardiovascular involvement, and those diagnosed with this disease have a high mortality rate. Therefore, any measure that can facilitate the early detection of cardiovascular injuries before the occurrence of symptoms can reduce mortality and morbidity in RA patients. RA has a high prevalence in middle-aged women who are especially at risk of cardiovascular problems and coronary involvement due to menopause. Therefore, early diagnosis of cardiovascular disorders can prevent the progress of associated damage and decrease the mortality rate in this population [1,4]. Given the limited number of studies on the use of the new diagnostic modalities, Doppler echocardiography and tissue Doppler imaging (TDI), in RA, the current study aimed to evaluate cardiovascular disorders in patients suffering from RA using these techniques.

\footnotetext{
* Corresponding Author: Nayyereh Saadati, E-mail: saadatin@mums.ac.ir, Tel: +98-51-38400001

Received: 08 December 2017; Accepted: 10 January 2018
} 


\section{Materials and Methods}

This descriptive, cross-sectional study was conducted on 40 RA patients [2-4] who referred to the Rheumatology Department and Clinic of Ghaem Hospital, affiliated with Mashhad University of Medical Sciences, Mashhad, Iran. RA was diagnosed based on the American College of Rheumatology criteria. In total, 40 patients with no clinical cardiovascular symptoms and normal electrocardiography (ECG), chest examination, and chest radiography results were entered into the study and investigated for six months. All patients were taking prednisolone, methotrexate, and hydroxychloroquine. Within this period, all necessary information was collected for further evaluation, and a complete rheumatology and cardiovascular assessment was carried out for each participant. Patients with a minimum of five years since their RA diagnosis and those without any cardiovascular diseases were included in the study. Exclusion criteria included a history of cardiac infarction or cerebrovascular problems (e.g., cerebrovascular accidents), any history of cardiac disease in the medical records, abnormal electrocardiography findings, and abnormal heart radiologic findings. Patients were subjected to electrocardiography, chest radiography, echocardiography (M-mode 2D), Doppler ultrasound, and tests of erythrocyte sedimentation rate, C-reactive protein, rheumatoid factor, and anti-cyclic citrullinated peptide. Subsequently, the subjects' cardiac information was recorded.

The initial cardiovascular examination of the patients included history taking, a complete physical examination, electrocardiography, and chest radiography. All participants were evaluated with transthoracic echocardiography and tissue Doppler imaging using the GE Vivid 3 Dimensions ultrasound system (GE Healthcare, USA) with a $2.5-3.5 \mathrm{MHz}$ probe in the left lateral decubitus position based on the guidelines of the American Society of Echocardiography and the European Association of Cardiovascular Imaging. All evaluations were recorded for further review. Different echocardiographic parameters were evaluated, including left ventricular systolic function, ejection fraction (EF), ventricular wall motion abnormalities, left ventricular diastolic function and its related parameters, early E-wave and end A-wave diastolic velocities, as well as E-wave deceleration time (DT). These estimations were accomplished by the placement of the Doppler probe on the lateral wall and interventricular septum and the assessment of the mean parameters of TDI, including systolic velocity $(\mathrm{Sm})$ as well as early- and end-diastolic velocities (Am).
Additionally, the right upper pulmonary vein blood flow was determined by the insertion of the Doppler probe on the vein, which also facilitated the estimation of systolic (S) and diastolic (D) wave velocities and reversed enddiastolic flow (Ar). Simultaneously, the left ventricular systolic and diastolic volumes, stenosis and insufficiency of each valve, pulmonary arterial pressure (PRP), tricuspid valve regurgitation velocity, the lack or presence of cardiomyopathy, and pericardial fluid were also assessed.

\section{Statistical analysis}

To analyze the data, values related to the echocardiographic variables, such as the diastolic, systolic, and TDI performance, end-systolic and diastolic volumes, as well as stenosis and insufficiency status of each valve, were entered into SPSS version 11.5. The demographic characteristics (e.g., age and gender) and other variables such as ECG and chest X-ray findings were also recorded. Moreover, PRP was entered as a quantitative variable and then added as a qualitative variable after considering the cut-off point of 30 . Data analysis was performed using the Chi-square, Fisher's exact, and Mann-Whitney U tests as well as the Pearson's correlation coefficient. The Kolmogorov-Smirnov test was applied to control the assumptions of parametric tests. A $p$-value less than 0.05 was considered statistically significant.

\section{Results}

In total, 40 patients with RA were evaluated in this study for cardiovascular conditions. According to the results, the mean age of the patients was $46.4 \pm 15.48$ years (age range: $20-80$ years). The evaluation of age distribution based on 10-year age groups revealed that the highest frequency was related to the age range of 30-39 years (Table 1). The most common valvular abnormality in the patients was insufficiency of the tricuspid and mitral valves. Out of 29 patients with tricuspid insufficiency, 1 , 1, and 27 subjects had severe, medium, and weak insufficiency, respectively. In addition, 20 out of 21 cases had weak mitral insufficiency, and one patient had medium regurgitation in this regard (Table 2). While 28 $(70 \%)$ subjects had normal diastolic cardiac function, 1 $(2.5 \%)$ and $11(27.5 \%)$ cases had grades II (pseudonormal) and I (loose defect) diastolic dysfunction, respectively. Neither grade III diastolic dysfunction (restrictive) nor left ventricular wall motion abnormalities were observed in any of the patients.

Echocardiographic evaluations revealed no cardiac wall motion abnormalities in the patients. The parameters 
related to mitral valve in Doppler echocardiography and TDI of the right upper pulmonary vein are presented in Table 3. The mean cardiac EF was $61.27 \pm 4.80$ with a minimum and maximum of 50 and 70 , respectively. In addition, the results of the Pearson's correlation coefficient were indicative of a significant positive relationship between $\mathrm{Sm}$ and cardiac EF ( $\mathrm{r}=0.398$, $P=0.011$ ). Regarding the relationship of TDI parameters with $\mathrm{A}$ and $\mathrm{E}$ mitral parameters and diastolic and systolic vein flows, the results of the Pearson correlation coefficient revealed no significant association between $\mathrm{Em}$ and $\mathrm{E}(\mathrm{r}=0.128, P=0.432)$. Moreover, no significant relationship was observed between Am and A ( $\mathrm{r}=0.032$, $P=0.844)$. However, there were direct correlations between Em and $\mathrm{D}(\mathrm{r}=0.589, P=0.001)$ as well as between $\mathrm{Sm}$ and $\mathrm{S}(\mathrm{r}=0.322, P=0.043)$. The investigation of the relationship between diastolic function and $\mathrm{EF}$ revealed that the mean EF values were $62.4 \pm 4.04$ and $58.3 \pm 5.64$ in the group with normal diastolic performance and patients with diastolic dysfunction, respectively.

Due to the non-normality of data distribution, the Mann-Whitney $U$ test was run to compare the two groups, and the results demonstrated a significant difference between in EF values of the study groups $(P=0.047)$. EF was significantly lower in the group with diastolic dysfunction compared with the group with normal diastolic function. The mean PRP was $72.53 \pm 5.73 \mathrm{mmHg}$, varying within $18-40 \mathrm{mmHg}$. Considering the cut-off point of $30 \mathrm{mmHg}$ for PRP, this measure was $<30 \mathrm{mmHg}$ in $24(60 \%)$ patients and $\geq 30$ $\mathrm{mmHg}$ in $16(40 \%)$ cases. Moreover, PRP level was significantly associated with age $(P=0.031)$. Those patients diagnosed with pulmonary hypertension were specifically older than those with normal PRP (Table 3).

Based on the results, there were no statistically significant differences in the frequency of various levels of frequency and diastolic dysfunction in age groups of below and above 45 years $(P=0.343)$.

Table 1. Frequency distribution of valvular disorders in patients with rheumatoid arthritis based on age groups

\begin{tabular}{|c|c|c|c|c|c|c|c|c|c|c|}
\hline \multirow{2}{*}{\multicolumn{2}{|c|}{$\begin{array}{c}\text { Type of valvular } \\
\text { disorder }\end{array}$}} & \multicolumn{2}{|c|}{ No disorder } & \multicolumn{2}{|c|}{ Weak disorder } & \multicolumn{2}{|c|}{ Medium disorder } & \multicolumn{2}{|c|}{ Severe disorder } & \multirow{2}{*}{ P-value } \\
\hline & & Frequency & $\%$ & Frequency & $\%$ & Frequency & $\%$ & Frequency & $\%$ & \\
\hline \multirow{2}{*}{$\begin{array}{c}\text { Aortic } \\
\text { insufficiency }\end{array}$} & $\geq 45$ & 19 & 86.4 & 3 & 13.6 & - & - & - & - & \multirow{2}{*}{0.565} \\
\hline & $<45$ & 15 & 83.3 & 3 & 16.7 & - & - & - & - & \\
\hline \multirow{2}{*}{ Mitral stenosis } & $\geq 45$ & 21 & 95.5 & - & - & 1 & 4.5 & - & - & \multirow{2}{*}{0.550} \\
\hline & $<45$ & 18 & 100.0 & - & - & - & - & - & - & \\
\hline \multirow{2}{*}{$\begin{array}{c}\text { Mitral } \\
\text { insufficiency }\end{array}$} & $\geq 45$ & 12 & 54.5 & 9 & 40.9 & 1 & 4.5 & - & - & \multirow{2}{*}{0.343} \\
\hline & $<45$ & 7 & 83.9 & 11 & 61.1 & - & - & - & - & \\
\hline \multirow{2}{*}{$\begin{array}{l}\text { Tricuspid } \\
\text { stenosis }\end{array}$} & $\geq 45$ & 21 & 95.5 & - & - & 1 & 4.5 & - & - & \multirow{2}{*}{0.550} \\
\hline & $<45$ & 18 & 100.0 & - & - & - & - & - & - & \\
\hline \multirow{2}{*}{$\begin{array}{c}\text { Tricuspid } \\
\text { insufficiency }\end{array}$} & $\geq 45$ & 9 & 40.9 & 12 & 54.5 & - & - & 1 & 4.5 & \multirow{2}{*}{0.057} \\
\hline & $<45$ & 2 & 11.1 & 15 & 83.3 & 1 & 5.6 & - & - & \\
\hline \multirow{2}{*}{$\begin{array}{l}\text { Pulmonary } \\
\text { insufficiency }\end{array}$} & $\geq 45$ & 21 & 95.5 & 1 & 4.5 & - & - & - & - & \multirow{2}{*}{0.423} \\
\hline & $<45$ & 16 & 88.9 & 2 & 11.1 & - & - & - & - & \\
\hline
\end{tabular}

Table 2. Frequency distribution of valvular disorders in patients with rheumatoid arthritis

\begin{tabular}{lc}
\hline \multicolumn{1}{c}{ Valvular disorder } & Frequency (Percentage value \%) \\
\hline Aortic stenosis & - \\
Aortic insufficiency & $6(15.0 \%)$ \\
Mitral stenosis & $1(2.5 \%)$ \\
Mitral insufficiency & $21(52.5 \%)$ \\
Tricuspid stenosis & $1(2.5 \%)$ \\
Tricuspid insufficiency & $29(72.5 \%)$ \\
Pulmonary stenosis & - \\
Pulmonary insufficiency & $3(7.5 \%)$ \\
\hline
\end{tabular}


Heart dysfunction in rheumatoid arthritis

Table 3. Results of Doppler echocardiography and tissue Doppler imaging in evaluation of parameters related to mitral valve and right upper pulmonary vein in patients with rheumatoid arthritis

\begin{tabular}{lcccc}
\hline Echocardiographic parameters & Mean & Standard deviation & Minimum & Maximum \\
\hline E & 86.55 & 24.45 & 45.0 & 165.0 \\
A & 78.15 & 16.56 & 52.0 & 110.0 \\
DT & 201.96 & 47.20 & 135.0 & 326.0 \\
S & 51.60 & 11.2 & 9.0 & 76.0 \\
D & 39.67 & 13.76 & 6.0 & 82.0 \\
Sm & 8.1 & 2.16 & 5.0 & 16.0 \\
Em & 9.4 & 3.44 & 4.6 & 19.0 \\
Am & 9.4 & 3.52 & 1.3 & 25.0 \\
\hline
\end{tabular}

Electrocardiographic and pulmonary radiographic findings were normal in all cases. The parameters of TDI, right upper pulmonary vein blood flow (A, D, S), and Doppler of blood flow of mitral valve (DT, A, and E) were evaluated through echocardiography (Figure 1). A comparison of the mentioned parameters indicated significant relationships between D and Em and between $\mathrm{Sm}$ and S. However, no significant association was observed between Am and A or between Em and E. The mean EF was 61.3 in the patients, and there was a significant relationship between this variable and $\mathrm{Sm}$. The diastolic function of patients was normal in $70 \%$ of cases; grades I and II dysfunctions were reported in $27.5 \%$ and $2.5 \%$ of the subjects, respectively.

A significant association was found between EF and diastolic dysfunction. EF was significantly reduced in patients with diastolic dysfunction. As the results indicated, the mean PRP was $27.35 \mathrm{mmHg}$. Considering the cut-off point of $30 \mathrm{mmHg}$, increased pulmonary hypertension was reported in $40 \%$ of patients. The most common valvular diseases were tricuspid and mitral valve regurgitation ( $72.5 \%$ and $52 \%$, respectively). In $70 \%$ of patients, diastolic function was normal, and there was a significant relationship between systolic function and diastolic abnormality. Pulmonary hypertension was detected in $40 \%$ of patients. Furthermore, a significant relationship was observed between $\mathrm{D}$ and Em as well as between $\mathrm{S}$ and $\mathrm{Sm}(P=0.001$ and $P=0.043$, respectively). However, no significant relationship was between $\mathrm{E}$ and Em or between $\mathrm{A}$ and $\mathrm{Am}(P=0.432$ and $P=0.844$, respectively).

\section{Discussion}

In this study, total of 40 RA patients with the mean age of $46.4 \pm 15.48$ years were investigated. The age group of 30-39 years had the highest frequency of cardiovascular complications. According to the literature, the risk of RA and its cardiovascular complications increases with age. This risk is particularly enhanced in women because of menopause, which provides the conditions for cardiovascular complications [2,5]. In the present study, $90 \%$ of the subjects were female. Females are also at a higher risk of RA $[1,3,6,7]$. As indicated in previous studies, the risk of RA is three times higher in female patients than male cases $[2,6]$. In. similar studies, such as that of Gonzalez which was conducted in Spain, the mean PRP was $30.3 \mathrm{mmHg}$ in RA patients, which was significantly higher than that of the control group. Additionally, $66 \%$ of the patients in the mentioned study had pulmonary hypertension $[1,8]$. Various studies have reported the high risk of diastolic dysfunctions, such as the study of John M. Davis III et al. [9] and that of Fawad Aslam et al. conducted in Texas [10].

In the present study, the risk of valvular disorders, especially tricuspid, mitral, and aortic insufficiency, was at a considerable level. Likewise, Lili Pan et al. (2016) reported the risk of tricuspid and aortic insufficiency as higher than that of other valvular disorders $[3,11,12]$. The risk of valvular disorders is significantly higher in patients with RA than in those without it [1]. The evaluation of the risk of valvular disorders across genders revealed no significant difference; however, this was expected due to the low number of male subjects in the present study. No specific relationship was observed between age and valvular disorders in the current study; accordingly, there was no significant difference between the age groups of $<45$ and $>45$ years in this regard. Nevertheless, the age group of $>45$ years had a higher risk of tricuspid, pulmonary, and aortic insufficiency, which are the main valvular abnormalities.

The increased risk of valvular disorders and cardiovascular problems with aging has been reported in various studies, including a research by Asus [13]. This might be due to the emergence of an inflammatory process in the form of chronic valvulitis as well as other underlying risk factors. The assessment of PRP across genders revealed no significant difference. However, the t-test results demonstrated a significant difference between patients with pulmonary hypertension and those without such condition in terms of age. The results 
further revealed that those patients with pulmonary hypertension were significantly older than those without such problem $(P=0.033)$ [11-13].

In addition, no significant difference was observed in the occurrence of diastolic dysfunction across genders; however, this result is not reliable because of the low number of male participants in this study. Patients with diastolic dysfunction were significantly older than those without such dysfunction. In another study performed in the United Kingdom, Aamer Sandoo evaluated the cardiovascular risk factors and the risks of cardiovascular, coronary artery, and cerebrovascular diseases as well as hypertension by assessing the clinical findings of 201 patients with RA. His results revealed that cardiovascular problems were associated with age. Sandoo focused on the effects of inflammation and classical cardiovascular disease risk factors on the vasculature and other causes, such as hypothyroidism, which predict the incidence of cardiovascular diseases in RA patients [14].

Another study reported no apparent association between cardiovascular diseases and the number of involved extra-articular organs, platelet count, hyperfibrinogenemia, or C-reactive protein level. However, the occurrence of cardiovascular problems was significantly associated with poor treatment response to medication for RA, and the recognized risk factors were considered as exacerbating factors. Therefore, these factors, along with the accurate use of COX-2 inhibitors and long-term application of high-dose corticosteroids, were considered as risk factors for cardiovascular diseases [5].

In line with the current findings, similar studies have reported that older individuals are at higher risk of cardiovascular complications caused by RA. This is relatively justifiable considering the risk of RA in women along with menopausal complications at older ages and the effect menopause has on the cardiovascular system.

This study was limited by the rather small sample size; therefore, caution in interpreting the results is recommended. Further studies can implement coronary calcium scans in determining future risk of cardiac events and assess disease activity and its correlation with cardiac abnormalities.

\section{Conclusion}

As the findings of the present study indicated, patients inflicted with RA are at significant risk for cardiovascular disorders, especially increased PRP, grade I diastolic dysfunction, and tricuspid, mitral, or aortic insufficiency. Furthermore, this risk was found to increase with aging.

\section{Conflicts of interest}

The authors declare no conflict of interest.

\section{References}

1. Assous N, Touzé E, Meune C, Kahan A, Allanore Y. Cardiovascular disease in rheumatoid arthritis: singlecenter hospital-based cohort study in France. Jt Bone Spine 2007; 74(1): 66-72. doi: 10.1016/j.jbspin.2006.10. 001.

2. Lazzerini PE, Capecchi PL, Laghi-Pasini F. Systemic inflammation and arrhythmic risk: lessons from rheumatoid arthritis. Eur Heart J 2016; 38(22): 1717-27. doi: 10.1093/eurheartj/ehw208.

3. Loegstrup B, Ellingsen T, Pedersen A, Kjaersgaard A, Botker H, Maeng M. P1081Incidence of heart failure and ischemic heart disease in patients with rheumatoid arthritis: A Danish population based study, 1995-2016. Eur Heart J 2017; 38 (suppl_1). doi: 10.1093/eurheartj/ ehx502.p1081. doi: 10.7860/JCDR/2014/8112.4349.

4. Mantel $\ddot{A}$, Holmqvist M, Andersson DC, Lund LH, Askling J. Association Between Rheumatoid Arthritis and Risk of Ischemic and Nonischemic Heart Failure. $\boldsymbol{J} \mathbf{A m}$ Coll Cardiol 2017; 69(10): 1275-85. doi: 10.1016/j.jacc. 2016.12.033.

5. Nissen SE, Yeomans ND, Solomon DH, Lüscher TF, Libby P, Husni ME, et al. Cardiovascular safety of celecoxib, naproxen, or ibuprofen for arthritis. $\boldsymbol{N}$ Engl J Med 2016; 375(26): 2519-29. doi: 10.1056/nejmoa 1611593.

6. Firestein GS, Budd R, Gabriel SE, McInnes IB, O'Dell JR.
Kelley and Firestein's Textbook of Rheumatology EBook: Elsevier Health Sciences; 2016.

7. Pincus T, Callahan L. Taking mortality in rheumatoid arthritis seriously-predictive markers, socioeconomic status and comorbidity. J Rheumatol 1986; 13(5): 841-5.

8. Al-Timimi DJ, Rasool MT, Sulaiman DM. HLA-DR/DQ genotypes in Kurd patients with rheumatoid arthritis: relation to disease activity. J Clin Diagn Res 2014; 8(5): CC01.

9. Davis JM, Lin G, Oh JK, Crowson CS, Achenbach SJ, Therneau TM, et al. Five-year changes in cardiac structure and function in patients with rheumatoid arthritis compared with the general population. Int J Cardiol 2017; 240: 379-85. doi: 10.1016/j.ijcard.2017.03.108.

10. Aslam F, Bandeali SJ, Khan NA, Alam M. Diastolic Dysfunction in Rheumatoid Arthritis: A Meta-Analysis and Systematic Review. Arthritis Care Res 2013; 65(4): 534-43. doi: 10.1002/acr.21861.

11. Khanna D, Gladue H, Channick R, Chung L, Distler O, Furst DE, et al. Recommendations for screening and detection of connective tissue disease-associated pulmonary arterial hypertension. Arthritis Rheumatol 2013; 65(12): 3194-201. doi: 10.1002/art.38172.

12. Sadeghi S, Granton JT, Akhavan P, Pasarikovski CR, Roos AM, Thenganatt J, et al. Survival in rheumatoid arthritis-associated pulmonary arterial hypertension 
Heart dysfunction in rheumatoid arthritis

compared with idiopathic pulmonary arterial hypertension. Respirology 2015; 20(3): 481-7. doi: 10.1111/resp.12464.

13. Prasad M, Hermann J, Gabriel SE, Weyand CM, Mulvagh $\mathrm{S}$, Mankad R, et al. Cardiorheumatology: cardiac involvement in systemic rheumatic disease. Nat Rev Cardiol 2015; 12(3): 168-76. doi: 10.1038/nrcardio.
2014.206.

14. Sandoo A, Chanchlani N, Hodson J, Smith JP, Douglas KM, Kitas GD. Classical cardiovascular disease risk factors associate with vascular function and morphology in rheumatoid arthritis: a six-year prospective study. Arthritis Res Ther 2013; 15(6): R203. doi: 10.1186/ $\operatorname{ar} 4396$. 